\title{
Clostridium difficile: Investigating Transmission Patterns Between Infected and Colonized Patients Using Whole Genome Sequencing
}

\begin{abstract}
Ling Yuan Kong, ${ }^{1}$ David W. Eyre, ${ }^{2,3}$ Jacques Corbeil, ${ }^{4}$ Frederic Raymond, ${ }^{4}$ A. Sarah Walker, ${ }^{3}$ Mark H. Wilcox ${ }^{5}$ Derrick W. Crook, ${ }^{2,6}$ Sophie Michaud, ${ }^{7}$ Baldwin Toye, ${ }^{8}$ Eric Frost, ${ }^{7}$ Nandini Dendukuri, ${ }^{9}$ Ian Schiller, ${ }^{10}$ Anne-Marie Bourgault, ${ }^{111}$ Andrew Dascal, ${ }^{12}$ Matthew Oughton, ${ }^{12}$ Yves Longtin, ${ }^{12}$ Louise Poirier, ${ }^{13}$ Paul Brassard, ${ }^{10}$ Nathalie Turgeon, ${ }^{14}$ Rodica Gilca, ${ }^{15}$ and Vivian G. Loo ${ }^{1}$

${ }^{1}$ Division of Infectious Diseases and Department of Medical Microbiology, McGill University Health Centre, Montréal, Québec, Canada; ${ }^{2}$ Nuffield Department of Medicine and ${ }^{3}$ National Institute for Health Research Oxford Biomedical Research Centre, John Radcliffe Hospital, United Kingdom; ${ }^{4}$ Centre de recherche CHUQ, Université Laval, Québec City, Québec, Canada; ${ }^{5}$ Department of Microbiology, Leeds Teaching Hospitals and University of Leeds, and ${ }^{6}$ National Infection Service, Public Health England, London, United Kingdom; and ${ }^{7}$ Department of Microbiology and Infectiology, Université de Sherbrooke, Québec, ${ }^{8}$ Division of Microbiology, Ottawa Hospital, University of Ottawa, Ontario, ${ }^{9}$ Technology Assessment Unit and ${ }^{10}$ Centre for Outcomes Research, Research Institute, McGill University Health Centre, ${ }^{11}$ Department of Microbiology, Centre Hospitalier de I'Université de Montréal, ${ }^{12}$ Division of Infectious Diseases, Jewish General Hospital, and ${ }^{13}$ Department of Microbiology, Hôpital Maisonneuve-Rosemont, Montréal, ${ }^{14}$ Department of Microbiology, Centre Hospitalier Universitaire de Québec, Hôtel-Dieu de Québec, and ${ }^{15}$ Québec Institute of Public Health, Québec City, Canada
\end{abstract}

\section{(See the Editorial Commentary by O'Hagan and McDonald on pages 210-2.)}

Background. Whole genome sequencing (WGS) studies can enhance our understanding of the role of patients with asymptomatic Clostridium difficile colonization in transmission.

Methods. Isolates obtained from patients with Clostridium difficile infection (CDI) and colonization identified in a study conducted during 2006-2007 at 6 Canadian hospitals underwent typing by pulsed-field gel electrophoresis, multilocus sequence typing, and WGS. Isolates from incident CDI cases not in the initial study were also sequenced where possible. Ward movement and typing data were combined to identify plausible donors for each CDI case, as defined by shared time and space within predefined limits. Proportions of plausible donors for CDI cases that were colonized, infected, or both were examined.

Results. Five hundred fifty-four isolates were sequenced successfully, 353 from colonized patients and 201 from CDI cases. The NAP1/027/ST1 strain was the most common strain, found in 124 (62\%) of infected and 92 (26\%) of colonized patients. A donor with a plausible ward link was found for 81 CDI cases (40\%) using WGS with a threshold of $\leq 2$ single nucleotide polymorphisms to determine relatedness. Sixty-five (32\%) CDI cases could be linked to both infected and colonized donors. Exclusive linkages to infected and colonized donors were found for 28 (14\%) and 12 (6\%) CDI cases, respectively.

Conclusions. Colonized patients contribute to transmission, but CDI cases are more likely linked to other infected patients than colonized patients in this cohort with high rates of the NAP1/027/ST1 strain, highlighting the importance of local prevalence of virulent strains in determining transmission dynamics.

Keywords. Clostridium difficile; CDI; whole genome sequencing; colonization.

Clostridium difficile is a leading cause of healthcare-associated diarrhea and a major cause of morbidity and mortality for hospitalized patients [1]. Patients with symptomatic infection and asymptomatic colonization are both known to shed spores into the environment [2]. Currently recommended infection control measures focus on the detection and isolation of symptomatic patients, believed to be responsible for most healthcare-associated transmission events [3]. However, recent molecular studies

Received 12 January 2018; editorial decision 10 April 2018; accepted 25 May 2018; published online May 28, 2018.

Correspondence: L. Y. Kong, Division of Infectious Diseases and Department of Medical Microbiology, McGill University Health Centre, 1001 Decarie Blvd, Montreal, Québec H4A 3J1, Canada (ling.kong@mail.mcgill.ca).

\section{Clinical Infectious Diseases ${ }^{\circledR} \quad$ 2019;68(2):204-9}

(C) The Author(s) 2018. Published by Oxford University Press for the Infectious Diseases Society of America. All rights reserved. For permissions, e-mail: journals.permissions@oup.com. DOI: 10.1093/cid/ciy457 using whole genome sequencing (WGS) have found that most new cases of $C$. difficile infection (CDI) in endemic settings could not be explained by transmission from symptomatic cases [4], raising interest in the role of colonized patients in transmission of $C$. difficile.

Typing methods used to identify transmission leading to CDI include pulsed-field gel electrophoresis (PFGE), polymerase chain reaction ribotyping, and multilocus sequence typing (MLST), among others [2]. With the advent of high-throughput sequencing technologies, WGS is increasingly being adopted as a preferred typing/fingerprinting method with high discriminatory power, and so has been used in multiple molecular epidemiology studies on C. difficile transmission [4-7]. In this study, using WGS of isolates and epidemiological data from a prospective cohort study, we aimed to elucidate the role of patients colonized with $C$. difficile in onward transmission of infection. 


\section{METHODS}

\section{Study Population and Definitions}

A multicenter prospective study was conducted between 6 March 2006 and 25 June 2007 to determine host and pathogen factors for healthcare-associated $C$. difficile infection and colonization, with results previously published [8]. In brief, data were collected in 6 Canadian, university-affiliated hospitals, on 15 study units (7 surgical units and 8 medical units). The selected units were those with a historically high or low incidence of CDI. All patients 18 years or older admitted to these hospital units were eligible for participation. Exclusion criteria included hemodynamic instability, palliative status, neutropenia (absolute neutrophil count $\leq 1000 / \mu \mathrm{L}$ ), or inability to participate in the informed-consent process.

Patients were followed daily until ward discharge, death, or withdrawal from the study. Rectal swabs or stool samples were obtained for culture on admission, weekly during hospitalization, and at onset of diarrhea (if applicable). Toxigenic C. difficile culture was performed on stool samples or rectal swabs using standard methods [9]. The cell cytotoxin neutralization assay was the diagnostic assay used in routine clinical care during the study period. Isolates were tested for presence of $t c d \mathrm{~A}$ and $t c d \mathrm{~B}$ using nucleic acid amplification methods $[10,11]$.

CDI was defined as the presence of diarrhea without an alternative explanation and a positive $C$. difficile cytotoxin assay or toxigenic culture, an endoscopic diagnosis of pseudomembranes, or a pathological diagnosis of CDI. Diarrhea was defined as at least 3 loose stools within at least one 24-hour period. Asymptomatic C. difficile colonization was defined as a positive stool C. difficile culture in the absence of diarrhea. Nontoxigenic strains of $C$. difficile were defined as culture positive and $t c d B$ negative.

To capture a more comprehensive picture of transmission, we also reviewed infection control data to determine the incidence of CDI cases in nonparticipants occurring on the study units during the study period. For one of the 6 participating hospitals, isolates were conserved for the purpose of infection control surveillance and were available for nonstudy incident CDI cases on study units; all incident CDI cases participated in the study for one other hospital. These isolates were included in the current analysis. Hospital and study unit admission and discharge dates were collected for every participant admitted to study units.

\section{Pulsed-field Gel Electrophoresis}

Each isolate underwent PFGE using standard methods [12] at the time of the study. Strain relatedness was determined using the criteria of Tenover et al using BioNumerics (Applied Maths) [13]. The Dice coefficient was used to measure similarity between patterns.

\section{DNA Preparation, Sequencing, Mapping, and Single-nucleotide Polymorphism Detection}

DNA was extracted using Purelink viral RNA/DNA minikit (Invitrogen, Burlington, Ontario, Canada) on a subcultured colony from frozen isolates. DNA was quantified using QuantiFluor dye (Promega). Sequencing libraries were prepared using the Nextera XT Sample Preparation Kit (Illumina, San Diego, California) with $1 \mathrm{ng}$ of purified DNA per sample. Dual indices were added during library preparation. Library concentrations were normalized using bead normalization as described by the manufacturer. Ninety-six libraries were pooled per HiSeq lane. Sequencing was performed on the HiSeq 2500 sequencer (Illumina) using v3 chemistry, generating paired-end 101-bp reads. Reads and assemblies have been deposited in the European Nucleotide Archive database in project PRJEB11776.

Sequence reads were analyzed and assembled using a previously described pipeline developed specifically for bacterial genomes [4]. The set of reads from each isolate was mapped using Stampy version 1.0.11 (without Burrows-Wheeler Aligner premapping, using an expected substitution rate of 0.01) [14] to the $C$. difficile 630 reference genome (GenBank: AM180355.1) [15]. Base-pair calls were identified across all mapped nonrepetitive core genome sites using SAMtools (version 0.1.19) mpileup with the extended base-alignment quality flag, using parameters based on bacterial sequences [4]. A consensus of $\geq 75 \%$ was required to support a nucleotide call, and calls were required to be homozygous under a diploid model. Only calls supported by $\geq 5$ reads, including one in each direction, were accepted.

Sequences were compared using single-nucleotide polymorphisms (SNPs), obtaining differences between sequences from maximum likelihood phylogenies constructed using PhyML [16] with generalized time-reversible substitution model and "BEST" tree topology search algorithm, corrected for the effect of recombination using ClonalFrameML [17] (with default settings). Sequence reads were also assembled de novo with Velvet [18] and MLSTs and toxigenic strains identified using BLAST (Basic Local Alignment Search Tool) searches of de novo assemblies ( $\geq 1000$ nucleotide identities with $t c d A$ or $t c d B$ genes).

\section{Transmission Analysis}

Isolates' PFGE, MLST, and toxigenic status were first examined according to colonized or infected status. Ward movement and WGS data were then combined to identify plausible donors for each CDI case. Proportions of plausible donors that were colonized or infected were calculated. Donors were identified for an isolate when they were determined to be clonal (differed by $\leq 2$ SNPs by WGS), and a plausible epidemiological link could be identified between the pair based on a previously described model [19], namely, the pair shared a ward after the donor tested positive and before the recipient tested positive, shared a ward before either tested positive, or if the recipient occupied a ward after the donor tested positive and was discharged. Maximum infectious period of 8 weeks, incubation period of 12 weeks, and ward contamination period of 26 weeks were allowed [20].

The analyses were first done for all available isolates, then restricted to 2 hospitals where $80 \%$ or more of all incident CDI cases occurring on study units during the study period were sequenced, whether part of the prospective study or not. 


\section{RESULTS}

Of 568 isolates from the cohort study, 513 were available for sequencing. An additional 52 isolates from 77 incident CDI cases from one of the participating hospitals were included for a total of 565 isolates. The participation rate in the initial prospective cohort study was $57.1 \%$ of eligible patients admitted to the study units. For one hospital contributing $9.6 \%$ of isolates, all incident CDI cases on study units were captured in the study. Figure 1 provides a breakdown of sample sources and patient statuses.

Overall, 554 (98\%) samples were sequenced successfully, from 550 patients ( 4 patients contributed 2 samples). There were 353 samples from colonized patients and 201 from infected patients. Two isolates did not have a PFGE pattern available, and 17 isolates could not be assigned to a known MLST.

The epidemic NAP1/ST1(ribotype 027) strain was the most commonly occurring strain among both infected and colonized patients, found in 124 (62\%) and 92 (26\%) patients, respectively. However, the majority of colonized patients carried strains from a variety of different sequence types (Figure 2). Strains from 27 different sequence types were found among infected patients, whereas a greater variety with 41 sequence types was found among colonized patients. The majority (74\%) of colonized patients carried toxigenic strains.

By comparing all samples from infected patients with prior samples from within the cohort, using a threshold of $\leq 2$ SNPs to determine relatedness, overall 105 (52\%) cases could be linked genetically to a prior sample (Table 1); 65 patients (32\%) could be linked to both infected and colonized donors. More cases were found to be related to isolates only from infected patients than isolates only from colonized patients (28 cases [14\%] and 12 cases [6\%]), respectively. Within all 105 cases related to a previous infected or colonized donor using WGS, a donor with

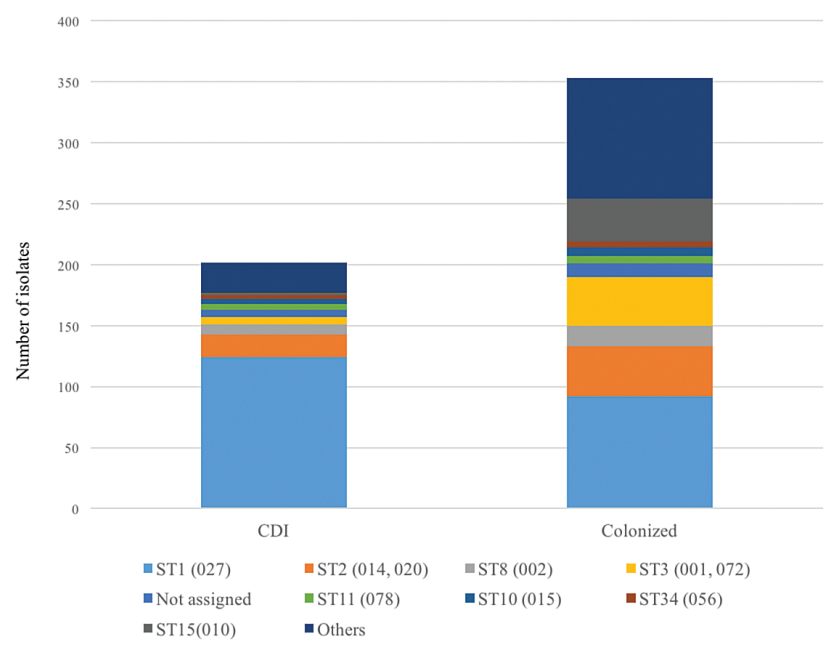

Figure 2. Multilocus sequence types by infected or colonized status. Polymerase chain reaction ribotypes are shown in parentheses. Abbreviations: CDI, Clostridium difficile infection; ST, sequence type.

a plausible ward link could be found for 81 patients (77\%; $40 \%$ of all 201 cases). Nearly all the identified donors were of the epidemic NAP1/ST1 strain. Only 7 patients with genetic and ward links were found to have non-NAP1/ST1 donors, including 3 linked to colonized donors only, 3 linked to infected donors only, and one to both infected and colonized donors.

Restricting analyses to the 2 hospitals with most complete data (Table 2), overall similar patterns were observed, including for those cases substantiated with ward links. Thirty of 117 cases $(26 \%)$ could be linked to isolates from both infected and colonized patients and $26(22 \%)$ to isolates from only infected patients, whereas only 4 (3\%) were linked to samples from only colonized patients. Of 46 cases with a ward link, 30 (26\% of all

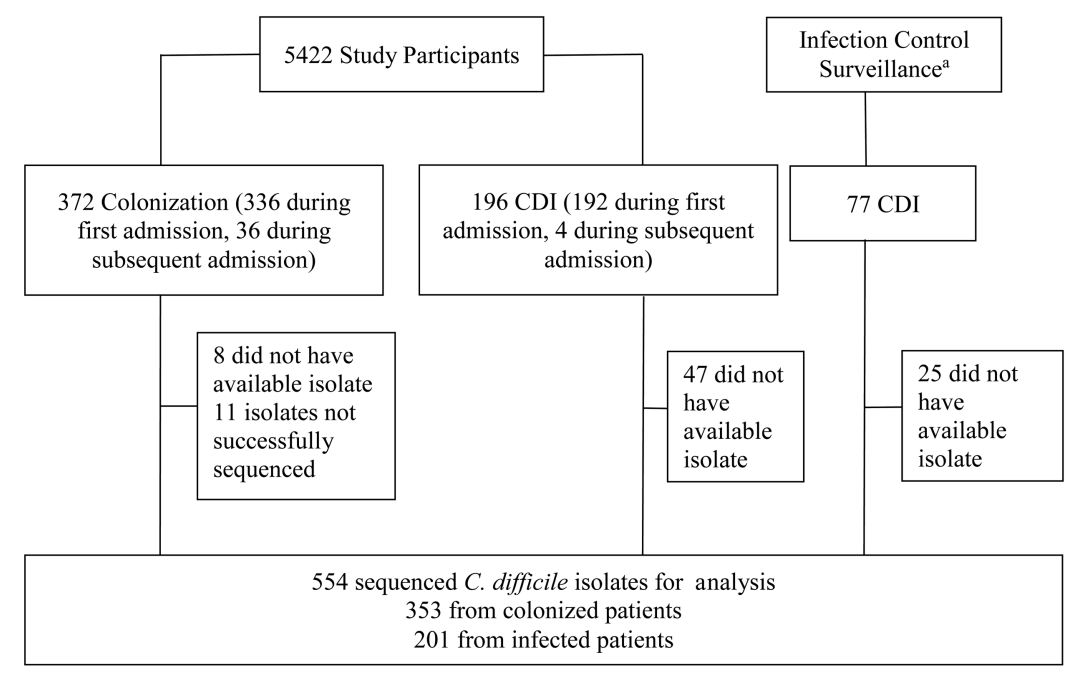

Figure 1. Flowchart of patients and isolates included in analysis. Infection control surveillance isolates were available for 1 site. Abbreviation: CDI, Clostridium difficile infection. 


\begin{tabular}{|c|c|c|c|c|}
\hline Possible Source & Genetically Linked & $\begin{array}{l}\text { NAP1/027/ST1 Among Genetically } \\
\text { Linked Donors }\end{array}$ & $\begin{array}{l}\text { Genetic and Ward } \\
\text { Link }\end{array}$ & $\begin{array}{c}\text { NAP1/027/ST1 Among Genetically and } \\
\text { Ward-linked Donors }\end{array}$ \\
\hline Linked to prior case & $105(52)$ & $95(91)$ & $81(40)$ & $74(91)$ \\
\hline Linked to infected patients only & $28(14)$ & $23(82)$ & $34(17)$ & $31(91)$ \\
\hline $\begin{array}{l}\text { Linked to colonized patients } \\
\text { only }\end{array}$ & $12(6)$ & $8(67)$ & $19(10)$ & $16(84)$ \\
\hline $\begin{array}{l}\text { Linked to both infected and } \\
\text { colonized patients }\end{array}$ & $65(32)$ & $64(99)$ & $28(14)$ & $27(96)$ \\
\hline
\end{tabular}

Data are presented as No. (\%) unless otherwise indicated.

117 cases) had an exclusive link to an infected donor, and only 2 ( $2 \%$ of all 117 cases) had an exclusive link to a colonized donor.

\section{DISCUSSION}

The role of colonized patients in transmission of CDI has been the subject of several previous molecular epidemiology studies $[7,19,21]$. Curry et al used multilocus variable number tandem repeats analysis genotyping and concluded that $29 \%$ of 56 incident CDI cases could be linked to colonized patients [21]. Using WGS, Eyre et al did not find evidence of any onward transmission from 18 asymptomatic colonized patients to CDI cases [19].

Using WGS, we investigated the contribution of colonized and infected patients in onward transmission toward incident CDI cases. In our larger cohort, $52 \%$ of cases could be linked to a previous patient. This is higher than previously reported rates [4], in part because our study includes both infected and colonized patients as sources, although higher linkage rates to symptomatic patients, 93 of 201 (46\%) cases, were also found. This difference may be explained in part by the diagnostic laboratory methods used. In the study by Eyre et al, the laboratory method used was immunoassay whereas in our study, the laboratory method was toxigenic culture, which has a higher sensitivity than enzyme immunoassay for detecting C. difficile. Therefore, more patients would have been classified as CDI and a higher linkage would be made with CDI patients. However, patients met the case definition for CDI and did not have an alternative explanation for diarrhea. In addition, the high incidence of CDI of 28.1 cases per 10000 patient-days in our cohort reflected the epidemic setting of the study, with a large pool of symptomatic patients, and a higher infection-to-colonization ratio compared to other cohorts [22]. The high proportion of infected patients is likely explained by the predominance of the NAP1/ST1 strain, which is more virulent and likely to cause infection [8].

Examining data from all units, an incident CDI case was 2.3 times more likely to be linked to an infected patient only than to a colonized patient only, whereas in the subset of hospitals with most complete data, this was 6 times more likely. Within the hospitals where data were most complete, exclusive linkage to colonized donors was less common; however, in these hospitals the proportion of infected cases sequenced (77\%-86\%) was substantially higher than on the other units $(26 \%-27 \%)$ due to availability of additional isolates. In both analyses, many cases could be linked to both infected and colonized patients, reflecting the outbreak setting in which the cohort study took place and the relatively slow rate of $C$. difficile evolution relative to the time between transmitted cases, enabling additional potential transmission links to be identified.

Our analyses suggest that colonized patients may be a source of onward transmission to incident CDI cases, but that spread from infected donors is likely more frequent. This could plausibly be explained by lower levels of shedding seen in colonized patients (without diarrhea) as compared with infected patients [23]. Onward transmission events from colonized individuals to infected patients in our cohort frequently carried the epidemic

Table 2. Proportions of Clostridium difficile Infection Cases Genetically and Epidemiologically Linked to Prior Infected and Colonized Donors Using Whole Genome Sequencing—2 Hospitals (117 Cases)

\begin{tabular}{|c|c|c|c|c|}
\hline Possible Source & Genetically Linked & $\begin{array}{c}\text { NAP1/027/ST1 Among Genetically } \\
\text { Linked Donors }\end{array}$ & $\begin{array}{l}\text { Genetic and Ward } \\
\text { Link }\end{array}$ & $\begin{array}{c}\text { NAP1/027/ST1 Among Genetically and } \\
\text { Ward-linked Donors }\end{array}$ \\
\hline Linked to prior case & $60(51)$ & $53(88)$ & $46(39)$ & $42(91)$ \\
\hline Linked to infected patients only & $26(22)$ & $21(81)$ & $30(26)$ & $27(90)$ \\
\hline $\begin{array}{l}\text { Linked to colonized patients } \\
\text { only }\end{array}$ & $4(3)$ & $3(75)$ & $2(2)$ & $2(100)$ \\
\hline $\begin{array}{l}\text { Linked to both infected and } \\
\text { colonized patients }\end{array}$ & $30(26)$ & $29(97)$ & $14(12)$ & $13(93)$ \\
\hline
\end{tabular}

Data are presented as No. (\%) unless otherwise indicated. 
NAP1/ST1 strain, possibly reflecting strain-specific characteristics, such as higher transmissibility [24] (increasing the chance of acquisition) and higher propensity to cause symptomatic infection and thereby increasing detection. For example, NAP1/ ST1 may be shed more profusely and persist more effectively in the environment. A study using WGS to track transmission similar to ours, but examining only ribotype-027 (NAP1/ST1) strains within one UK hospital, found that $60 \%$ of their genetically related strains were circulated by ward-based contamination [7]. However, another possibility for the greater degree of linkage is the relatively recent emergence of this fluoroquinolone-resistant NAP1/ST1, resulting in less population-wide genetic diversity, and thus increasing the chance of observing genetic linkage without direct transmission.

The limitations in our study include the incomplete sampling in the participating hospitals. Overall, we only obtained fecal samples from $57 \%$ of eligible participants, and did not capture all CDI cases on all study units. Incomplete sampling leads to the proportion of linked cases being underestimated as some potential transmission donors are missed. Patients who were ineligible in the initial cohort study represent another pool of potential missed linkages, since previously determined eligibility criteria (eg, neutropenia) for the prospective study do not necessarily translate to a ward-based transmission analysis study. Ideally, studies focused on ward-based transmission would be less restrictive, given the very low risk posed to patients of undergoing rectal swabs. Increased participation could have been achieved by waiving written informed consent and obtaining verbal consent and implementation or wardbased communication tools explaining the option to opt out.

When limiting the analyses to 2 hospitals with $>80 \%$ incident cases contributing isolates for sequencing, rates of linkage to infected patients increased, but this could represent sampling bias given that more infected donors were available. Finally, although all transmission events were inferred from the genetic data, other sources, such as patients not included in analyses, including ineligible patients, and the environment were not sampled and may be other reservoirs of $C$. difficile leading to CDI.

Our study provides new insight into the epidemiology of transmission between colonized and infected patients, by deriving data from the largest cohort to date of colonized and infected patients along with geographic ward information. We also confirm the utility of WGS in conjunction with epidemiological data to track transmission, which is increasingly studied including in healthcare epidemiological models.

\section{CONCLUSIONS}

Patients colonized with C. difficile without diarrhea contribute to the transmission of infection, but more transmission events appear to originate from infected patients with diarrhea.
Certain strains, such as the epidemic NAP1/ST1 strain, may be more transmissible and virulent, and hence more likely to cause more symptomatic infection following contact with infected and asymptomatically colonized patients. Thus, the relative contribution of colonized and infected patients toward onward transmission is likely dependent on the local prevalence of virulent strains.

\section{Supplementary Data}

Supplementary materials are available at Clinical Infectious Diseases online. Consisting of data provided by the authors to benefit the reader, the posted materials are not copyedited and are the sole responsibility of the authors, so questions or comments should be addressed to the corresponding author.

\section{Notes}

Acknowledgments. We are grateful to Dr Marcel Behr and Dr Robyn Lee for their help and insightful discussions.

Disclaimer. The funding sources did not have any role in the design of the current study, its analysis, interpretation of data, or writing of the report.

Financial support. This work was supported by the funding source of the prospective cohort study, the Consortium de Recherche sur le Clostridium difficile. The partners of the Consortium include: Fonds de la recherche en santé du Québec; Canadian Institutes of Health Research; Ministère de la Santé et des Services sociaux du Québec; Institut national de santé publique du Québec; Health Canada; Centre hospitalier de l'Université de Montréal; McGill University Health Centre; Centre hospitalier universitaire de Québec; and Centre hospitalier universitaire de Sherbrooke.

Potential conflicts of interest. M. H. W. reports grants and personal fees from Actelion, Cubist, Astellas, Merck, Sanofi-Pasteur, Summit, Seres, bioMérieux; and personal fees from Valneva, Alere, Ferring, and Synthetic Biologics. He reports personal fees from AstraZeneca, Nabriva, Pfizer, Roche, The Medicines Company, Basilea, Bayer, Allergan, Menarini, Motif Biosciences, AiCuris, Antabio, and Spero; and grants and personal fees from Abbott, European Tissue Symposium, Paratek, Tetraphase, and Surface Skins. D. W. C. reports grants from Wellcome Trust and Medical Research Council (UK) and National Institute for Health Research (UK). A. D. has received grants from Fonds de la recherche en santé du Québec and Canadian Institutes of Health Research. M. O. reports grants and personal fees from Merck, and grants from Sanofi and Summit. Y. L. reports grants from Merck and Becton Dickinson. L. P. reports grants from Consortium de recherché sur le Clostridium difficile. N. T. reports grants from Consortium de recherche sur le Clostridium difficile. She reports payment for lectures from Fédération des médecins omnipraticiens du Québec and Pendopharm. R. G. reports grants from Sanofi Pasteur and Pfizer. V. G. L. reports grants from Consortium de recherche sur le Clostridium difficile, and consultancy fees and payment for lectures from Merck. All other authors report no potential conflicts. All authors have submitted the ICMJE Form for Disclosure of Potential Conflicts of Interest. Conflicts that the editors consider relevant to the content of the manuscript have been disclosed.

\section{References}

1. Evans CT, Safdar N. Current trends in the epidemiology and outcomes of Clostridium difficile infection. Clin Infect Dis 2015; 60(Suppl 2):S66-71.

2. Martin JS, Monaghan TM, Wilcox MH. Clostridium difficile infection: epidemiology, diagnosis and understanding transmission. Nat Rev Gastroenterol Hepatol 2016; 13:206-16.

3. Cohen SH, Gerding DN, Johnson S, et al; Society for Healthcare Epidemiology of America; Infectious Diseases Society of America. Clinical practice guidelines for Clostridium difficile infection in adults: 2010 update by the Society for Healthcare Epidemiology of America (SHEA) and the Infectious Diseases Society of America (IDSA). Infect Control Hosp Epidemiol 2010; 31:431-55.

4. Eyre DW, Cule ML, Wilson DJ, et al. Diverse sources of C. difficile infection identified on whole-genome sequencing. N Engl J Med 2013; 369:1195-205.

5. Didelot X, Eyre DW, Cule M, et al. Microevolutionary analysis of Clostridium difficile genomes to investigate transmission. Genome Biol 2012; 13:R118. 
6. Eyre DW, Golubchik T, Gordon NC, et al. A pilot study of rapid benchtop sequencing of Staphylococcus aureus and Clostridium difficile for outbreak detection and surveillance. BMJ Open 2012; 2. doi:10.1136/bmjopen-2012-001124.

7. Kumar N, Miyajima F, He M, et al. Genome-based infection tracking reveals dynamics of Clostridium difficile transmission and disease recurrence. Clin Infect Dis 2016; 62:746-52.

8. Loo VG, Bourgault AM, Poirier L, et al. Host and pathogen factors for Clostridium difficile infection and colonization. N Engl J Med 2011; 365:1693-703.

9. Clabots CR, Gerding SJ, Olson MM, Peterson LR, Gerding DN. Detection of asymptomatic Clostridium difficile carriage by an alcohol shock procedure. J Clin Microbiol 1989; 27:2386-7.

10. Spigaglia P, Mastrantonio P. Molecular analysis of the pathogenicity locus and polymorphism in the putative negative regulator of toxin production $(\mathrm{TcdC})$ among Clostridium difficile clinical isolates. J Clin Microbiol 2002; 40:3470-5.

11. Gonçalves C, Decré D, Barbut F, Burghoffer B, Petit JC. Prevalence and characterization of a binary toxin (actin-specific ADP-ribosyltransferase) from Clostridium difficile. J Clin Microbiol 2004; 42:1933-9.

12. Fawley WN, Wilcox MH. Pulsed-field gel electrophoresis can yield DNA fingerprints of degradation-susceptible Clostridium difficile strains. J Clin Microbiol 2002; 40:3546-7; author reply 3547.

13. Tenover FC, Arbeit RD, Goering RV, et al. Interpreting chromosomal DNA restriction patterns produced by pulsed-field gel electrophoresis: criteria for bacterial strain typing. J Clin Microbiol 1995; 33:2233-9.

14. Lunter G, Goodson M. Stampy: a statistical algorithm for sensitive and fast mapping of Illumina sequence reads. Genome Res 2011; 21:936-9.
15. Sebaihia M, Wren BW, Mullany P, et al. The multidrug-resistant human pathogen Clostridium difficile has a highly mobile, mosaic genome. Nat Genet 2006; 38:779-86.

16. Guindon S, Gascuel O. A simple, fast, and accurate algorithm to estimate large phylogenies by maximum likelihood. Syst Biol 2003; 52:696-704.

17. Didelot X, Wilson DJ. ClonalFrameML: efficient inference of recombination in whole bacterial genomes. PLoS Comput Biol 2015; 11:e1004041.

18. Zerbino DR, Birney E. Velvet: algorithms for de novo short read assembly using de Bruijn graphs. Genome Res 2008; 18:821-9.

19. Eyre DW, Griffiths D, Vaughan A, et al. Asymptomatic Clostridium difficile colonisation and onward transmission. PLoS One 2013; 8:e78445.

20. Walker AS, Eyre DW, Wyllie DH, et al. Characterisation of Clostridium difficile hospital ward-based transmission using extensive epidemiological data and molecular typing. PLoS Med 2012; 9:e1001172.

21. Curry SR, Muto CA, Schlackman JL, et al. Use of multilocus variable number of tandem repeats analysis genotyping to determine the role of asymptomatic carriers in Clostridium difficile transmission. Clin Infect Dis 2013; 57:1094-102.

22. Longtin Y, Paquet-Bolduc B, Gilca R, et al. Effect of detecting and isolating Clostridium difficile carriers at hospital admission on the incidence of $C$. difficile infections: a quasi-experimental controlled study. JAMA Intern Med 2016; 176:796-804.

23. Donskey CJ, Kundrapu S, Deshpande A. Colonization versus carriage of Clostridium difficile. Infect Dis Clin North Am 2015; 29:13-28.

24. Eyre DW, Fawley WN, Rajgopal A, et al. Comparison of control of Clostridium difficile infection in six English hospitals using whole-genome sequencing. Clin Infect Dis 2017; 65:433-41. 